\title{
Assessment of the Effects of Manual Chest Compression Technique on Atelectasis in Infants: A Randomized Clinical Trial
}

\author{
Natania Florentino Diniz'1, Evelim Leal de Freitas Dantas Gomes' \\ Cristiane Aparecida Moran', Silvana Alves Pereira², \\ Livia Maria de Andrade Martins' ${ }^{1}$, Luciana Carnevalli Pereira ${ }^{1}$ \\ ${ }^{1}$ Universidade Nove de Julho (UNINOVE), São Paulo, Brazil \\ ${ }^{2}$ Universidade Federal do Rio Grande do Norte (UFRN/FACISA), Santa Cruz, Brazil \\ Email: evelimgomes@uninove.br
}

Received 1 February 2014; revised 1 March 2014; accepted 31 March 2014

Copyright (C) 2014 by authors and Scientific Research Publishing Inc.

This work is licensed under the Creative Commons Attribution International License (CC BY). http://creativecommons.org/licenses/by/4.0/

(c) (i) Open Access

\begin{abstract}
Objectives: To evaluate the effect of manual chest compression (MCC) in the variables of oxygenation, hemodynamic and respiratory effeteness in infants suffering from respiratory diseases with atelectasis. Methods: Controlled clinical trial, in which 38 infants were evaluated, 19 in each study group (group A: atelectasis and group B: control). Data were measured before, immediately after and 10 minutes after the end of the technique's application. Results: The average age was of 5.05 months. There was an increase of RR in group A immediately after the application of the technique and signs of respiratory distress with a decrease in the oxygen saturation. Conclusion: There was a reduction in $\mathrm{SpO}_{2}$, an increase of $\mathrm{RR}$ and a worsening of clinical signs of respiratory distress. Given this, one may consider that there is a controversy about the benefits, mechanism of action, physiological and therapeutic effects of MCC when applied to infants.
\end{abstract}

\section{Keywords}

Pulmonary Atelectasis, Physiotherapy Modality, Respiratory Therapy, Infant; Infant Care, Clinical Trials

\section{Introduction}

Every year, around the world, 15 million children under 5 years old die from respiratory diseases, especially pneumonia (PNA) and Acute viral bronchiolitis (AVB) [1]. 
The consequences, medium and long term, of infections of the lower respiratory tract in children, especially the involvement of viral features, are of structural and functional order [2]. Between respiratory infections in early childhood, AVB [3] and PNA [4] have the most deleterious effects and may lead to some complications. Among the most common complications, atelectasis [5], when present, reveals a severity in the course of the disease.

Chest physical therapy is indicated for the treatment of many respiratory diseases, acting in patients requiring bronchial hygiene, respiratory rehabilitation or during the pre- and postoperatively [2]. Their objectives consist in the presence or reduction of the impact of obstruction by secretion, such as hyperinflation, atelectasis, misdistribution of ventilation, changes in ventilation/perfusion (V/P) and increased efforts to breathe [2].

Although widely used in hospitals, the techniques used in respiratory physiotherapy present few studies with well-defined and structured methods in pediatrics that allow comparisons by meta-analysis [6].

The targeting technique of the flow, also known as manual chest compression (MCC) or unilateral chest compression is a feature of respiratory therapy that is widely used and consists of the compression of the hemithorax with normal ventilation, to increase the air flow to the hypoventilated hemithorax, but there need to be criteria for its use especially in the pediatric population.

The study made by Sixel et al. [7] aimed to characterize the respiratory pattern before, during and after the unilateral chest compression in patients with an artificial airway, and during the technique obtained as a result an increase in the respiratory rate (RR), reduction in tidal volume (TV) and maintenance of minute volume (MV) when compared to before the intervention. They concluded that unilateral chest manual compression leads to changes in the respiratory pattern in the artificial airway and in spontaneous ventilation.

It is assumed that the therapeutic use of MCC as an adjuvant in respiratory therapy promotes respiratory changes. However, there is a need for more clinical studies, with infants who present pulmonary infections and evolve with atelectasis, in order to better clarify its use and effects. The hypothesis is that through the increased thoracic complacence of infants the effect of this technique is understated and not much effective for this age group justifying the need for this study in order to establish criteria for the use of MCC.

The aim of this study was to evaluate the effect of the manual chest compression technique in oxygenation variables, hemodynamic and respiratory activity in infants suffering from respiratory diseases with atelectasis.

\section{Materials and Methods}

This research was conducted after the approval of the UNINOVE Research Ethics Committee (protocol $\mathrm{n}^{0}$. 40325/2012) and after the knowing and authorization of the person responsible for the unit (nursery) involved in the study. Testing was registered in clinicaltrials.gov under registration number NCT01858844.

The subjects involved in the research were identified only by numbers and their anonymity was maintained. To participate in the study, all children had the consent of a parent or legal guardian signed by writing.

\subsection{Study Design}

A prospective study was made, by clinical trial, randomized, controlled, conducted in the nursery of the Mandaqui Hospital in the city of São Paulo.

Infants were eligible for the study (29 days old to 24 months old). After the patients' legal guardian read, agreed and signed the informed consent, the patients were enrolled into 2 groups. In group A were included patients diagnosed with atelectasis, by the medical staff, through thorax X-ray with signs of homogeneous opacification and whose underlying disease was respiratory. In group B (control group) were included children whose admission were not because of respiratory diseases and did show any signs of pain at the time of evaluation. For the control group, were used the same evaluation criteria as those from group A.

Infants with respiratory problems that were not atelectasis and infants who showed signs of irritability or intense crying during evaluation were excluded from the study (Flowchart according to Consort Statment 2010). The evaluator was blind to the child's diagnosis and therefore unaware of the group to which it belonged (Figure 1).

\subsection{Data Collection}

During the time of collection patients had been at least 12 hours without going through respiratory therapy. 


\section{Flowchart}

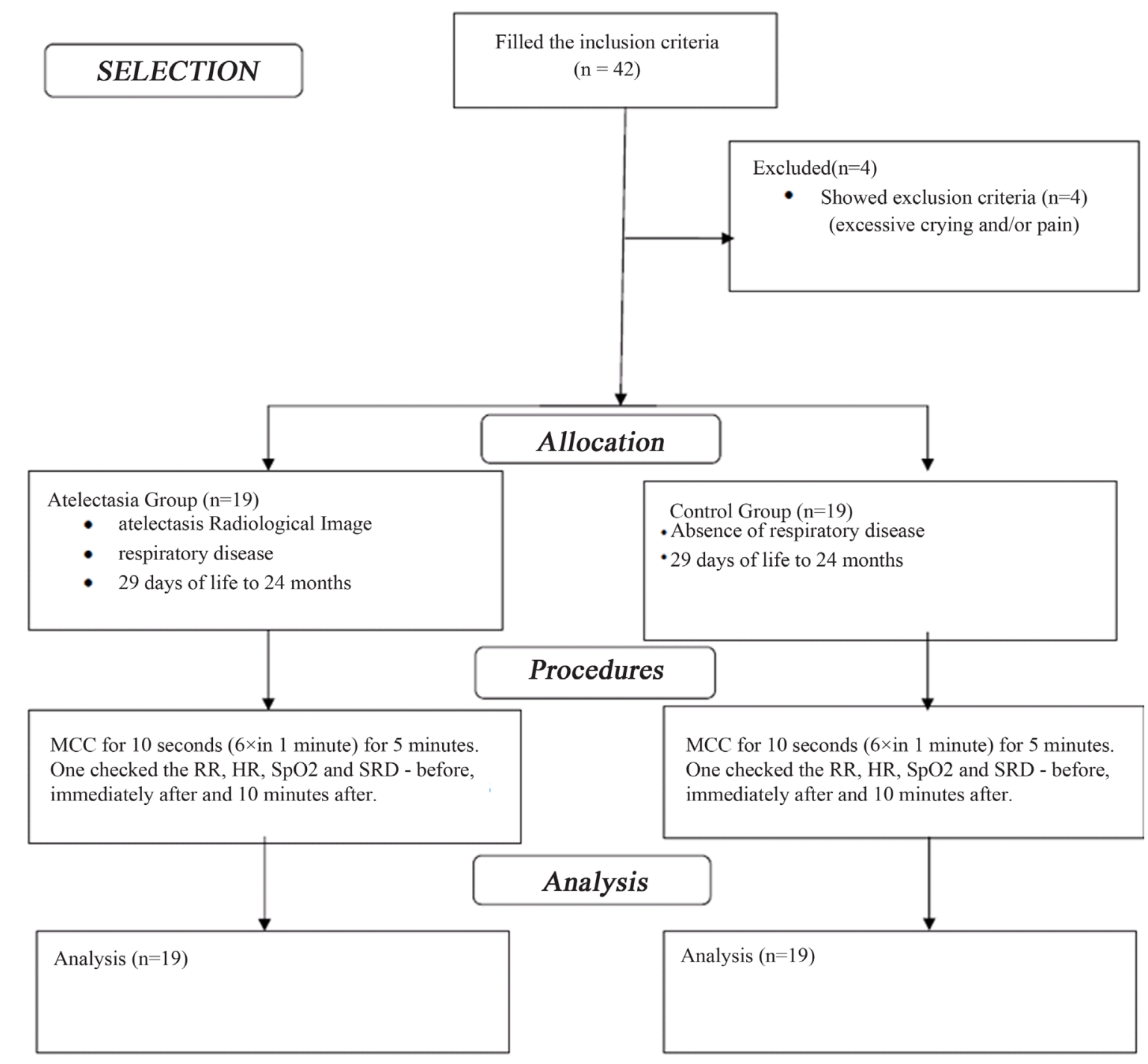

\section{Figure 1. Consort statement flowchart.}

The MCC was performed only once. Before the beginning of data collection the patients were placed in supine position, with the slope of the headboard at 30 degrees for the collection of signs of respiratory distress (SRD) (drawings), Respiratory rate (RR) (timed for 1 minute), heart rate (HR) and $\mathrm{SpO}_{2}$ through the Oximeter Pulse of the Clinical Guard ${ }^{\mathrm{TM}}$ brand.

\subsection{Procedure}

The MCC was performed with the placement of a hand on the hemithorax that was not affected. Following was applied a symmetric pressure of oblique direction, from top to bottom on the hemithorax so that it would shift according to the complacency of the rib cage. This pressure was applied during the expiration phase and held for 10 seconds, totaling six repeats of the maneuver for 60 seconds. The other hand held support in the contralateral side of the iliac crest to stabilize the patient. The technique was performed for 5 consecutive minutes with an interval of a respiratory excursion every 60 seconds. The data were checked again immediately after and 10 minutes after the application of the technique. 


\section{Statistical Analysis and Sample Estimation}

For statistical analysis was used the adherence to the curve test of Gaussian Kolmogorov-Smirnov (KS), the values are expressed in average and standard deviation because they have shown a normal distribution. For the evaluation of the variables during the three moments, was used ANOVA one-way, for comparison between groups was used the unpaired t test and for the correlation was used the Pearson test. A statistical significance at p $<0.05$ was considered. The softwares used were Instat and Minitab 14.

Amostral calculation was done considering an alpha error of 0.05 and a power of $80 \%$ based on the study's primary outcome which is the evaluation of hemodynamic and respiratory effects of the manual chest compression technique with an expected difference of at least $5 \%$, as described in the literature. Thus was obtained $\mathrm{n}=$ 38 subjects.

\section{Results}

42 children were evaluated, 4 were excluded, were evaluated 19 children with diseases based on respiration who developed a diagnosis associated with atelectasis (group A) and 19 children without diseases based on respiration (group B); aged between 29 days and 24 months were included. There was no discrepancy in relation to age groups, i.e., the difference in age between the groups and intra-groups was homogeneous (5.05 months).

Regarding groups A (atelectasis) and B (control), the values of HR, RR and SpO2 before, after and 10 minutes after the maneuver of MCC are shown in Table 1 expressed in average and standard deviation.

There was no statistical difference between the groups at the first moment despite the SpO2 in group B being greater than in group A (remembering that group B had no respiratory impairment). The MCC caused an increase of RR in group A immediately after and it was higher than the pre RR and higher than B's RR at the same time of evaluation.

There was a decrease in SpO2 in group A compared to B immediately after and 10 minutes after the application of MCC.

The classification of signs of respiratory distress (SRD) is given as follows: grade 1 was assigned to any infant who suffered intercostal retractions, grade 2 for infants who had retraction of the sternal notch and grade 3 for those infants who had more than 2 SRD.

Group A (atelectasis) after MCC evolved with an increase of SRD. Although this difference was not statistically significant it is worth noting that an increase in SRD should always be taken into consideration as it is an important parameter in the assessment and clinical development of the infant. Group B (control) did not show any RDS on any of the three moments of evaluation. (Table 1)

A positive correlation between $\mathrm{SpO}_{2}$ and $\mathrm{HR}$ was found in group A immediately after the MCC. (Figure 2)

\section{Discussion}

The technique of manual chest compression (MCC) is rarely described in the literature [7] in both children and

Table 1. Assessed clinical signs pre and post MCC.

\begin{tabular}{|c|c|c|c|c|c|c|}
\hline & \multicolumn{3}{|c|}{ Atelectasis $G$ roup $(n=19)$} & \multicolumn{3}{|c|}{ Control Group $(n=19)$} \\
\hline & HR & $\mathrm{SpO}_{2}$ & $\mathbf{R R}$ & HR & $\mathrm{SpO}_{2}$ & $\mathbf{R R}$ \\
\hline Before & $142.4 \pm 21.5$ & $95.7 \pm 3.0$ & $46 \pm 12.8$ & $138.7 \pm 22.2$ & $97.2 \pm 1.5$ & $46.4 \pm 9.3$ \\
\hline After & $144.3 \pm 23.9$ & $95.1 \pm 3.3^{\#}$ & $55.1 \pm 18.3^{* \#}$ & $141.1 \pm 26.8$ & $97.8 \pm 0.9$ & $45.5 \pm 10.0$ \\
\hline \multirow[t]{2}{*}{$10 \mathrm{~min}$. After } & $142.1 \pm 23.7$ & $94.3 \pm 4.2^{\#}$ & $46.21 \pm 15.6$ & $143.3 \pm 21.1$ & $98.0 \pm 0.7$ & $49.0 \pm 9.2$ \\
\hline & \multicolumn{3}{|c|}{ Signs of Respiratory Distress (SRD) } & \multicolumn{3}{|c|}{ Signs of Respiratory Distress (SRD) } \\
\hline Before & \multicolumn{3}{|c|}{0} & \multicolumn{3}{|c|}{0} \\
\hline After & \multicolumn{3}{|c|}{2} & \multicolumn{3}{|c|}{0} \\
\hline $10 \mathrm{~min}$. After & \multicolumn{3}{|c|}{2} & \multicolumn{3}{|c|}{0} \\
\hline
\end{tabular}

HR: heart rate, RR: respiratory rate, $\mathrm{SpO}_{2}$ oxygen saturation, ${ }^{*} \mathrm{p}<0.05$ in the intragroup comparison, ${ }^{*} \mathrm{p}<0.05$ in intergroup comparison. 


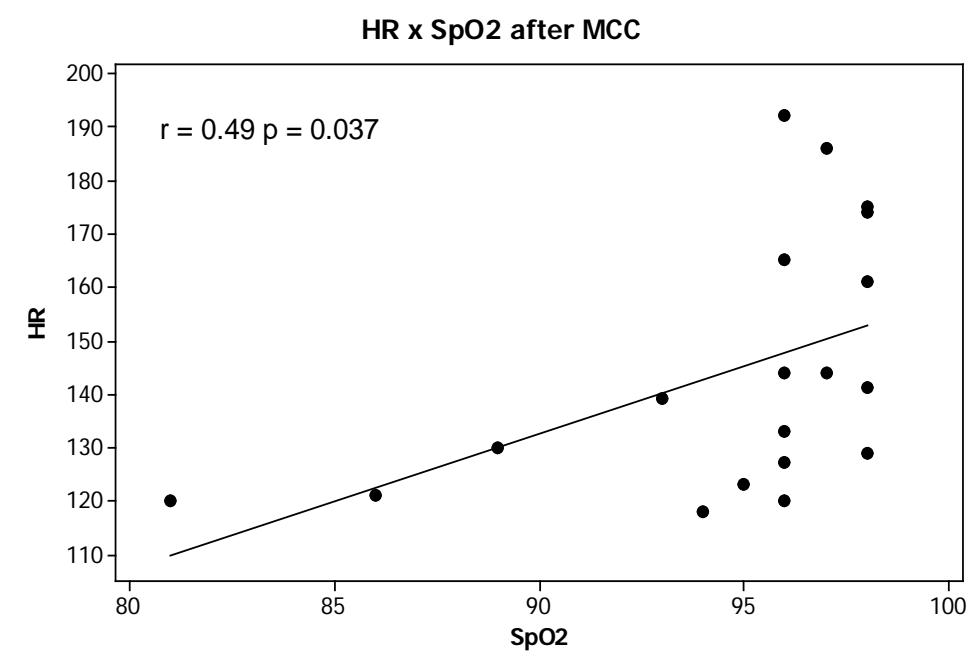

Figure 2. Correlation between $\mathrm{HR}$ and $\mathrm{SpO}_{2}$ in the Atelectasis group immediately after the MCC maneuver.

adults. Our findings show an increase in respiratory frequency and SRD with reduced oxygen saturation after the application of the MCC technique.

Lima et al. [8] conducted a research where the goal was to evaluate the effectiveness of the technique of flow directing (DF) also known as MCC in Wistar rats. 24 rats were divided into 4 groups (control, atelectasis, group treated with 3 chest compressions (T3) and the group treated with 6 chest compressions (T6) ), being the Atelectasis group, T3 and T6 induced to develop alveolar collapse. The chest compression technique was performed for 5 seconds, on the right hemithorax, directing the flow to the left lung. The results show that the amount of collapsed alveoli of the atelectasis group, T3 and T6 showed no changes among themselves, however, compared with the alveoli of the control group did not show improvement on the left side. The right side (the one that received compression) did not show significant changes in any of the four groups.

Atelectasis are frequent pulmonary changes in intensive care units and postoperative recovery rooms, it may determine worsening of the children's symptoms with predisposition to infectious complications and need for increased ventilatory support (oxygen therapy, invasive mechanical ventilation (MV) and/or noninvasive). They must be prevented, recognized and treated to prevent its complications and consequent morbidity [5].

Until about the second year of age, interductal and interalveolar communications (pores of Kohn and channels of Lambert) are practically inexistent. These structures are important because they allow collateral ventilation of alveoli units in case of obstruction of a bronchus or terminal bronchiole. Their absence explains the high prevalence of collapses and microatelectasis found in the respiratory diseases of young children [2] [3] [5].

The lung re-expansion is able to prevent or reverse ventilatory inequality situations, through the variation of intrathoracic pressures or by proper positioning, assisting in the optimization of pulmonary perfusion. The maneuver of thoracic blockade or MCC is indicated in patients that are less cooperative [7]-[9], but its effects are questionable and poorly studied.

In another study, Herri [10] conducted a survey with 16 children, with an average age of 10 days, with a diagnosis of atelectasis who were on mechanical ventilation or non-invasive ventilation, where the technique called "technique of insufflatory exercise in atelectasis" (TILA) was applied. It consisted of manually compressing all of the healthy lungs' territories during expiration through compression. Only the area with atelectasis was left clear and all children were in invasive or noninvasive mechanical ventilation. The results were obtained by using the radiological analysis before and after the technique, and demonstrated a success rate of 93.15\% in the atelectasis resolution. Herri believes that TILA had no adverse effect. The high rate of success can be explained by the fact that the children were ventilated with positive pressure, an important factor to compensate for the mechanical distortion between the lung and the ribcage in infants.

Della Via et al [9] applied the MCC associated with decompression in adults under MV and observed positive effects in the minute volume, tidal volume and oxygenation proving that the improvement attributed to the technique occurs in patients with positive pressure on airways. 
The difference between these two techniques (MCC and TILA) can be explained if we assume the representation of the respiratory system. The same can be described as a linear mechanical system based on the theoretical concept stated by Röher in 1915. This equation can be represented as follows: $\mathrm{P}=\Delta \mathrm{V}^{\prime} \mathrm{x} R+\Delta \mathrm{Vx}$ E, where $\mathrm{P}$ is the pleural pressure, $V$ ' the flow, $R$ is the resistance, $V$ the volume and $E$ the elastance [11]. The first part of the equation where the physical quantities of resistance and flow are expressed is related to the conducting airways (bronchial) and the second part where the volume change is related to elastance relates to the alveolus.

So if we consider that the technique of blocking is to change the flow, we will be acting in the airway of conduction without however reaching the parenchyma of the lung, as well as producing an increase in airway resistance by reducing its diameter as well as the diameter of the ribcage by compression maintained on these highly compliant structures [12].

Therefore, a change in volume would be necessary to reach the collapsed alveoli. This effect can be achieved during the course of TILA since this technique uses the combination of positive pressure, producing a greater alveolar pressure, an increase in functional residual capacity (FRC) and therefore recruitment of alveoli units in this region. This mechanical concept explains the success rate achieved by Herri [10] in his study.

Another important mechanical issue is that in the passive expiration of an adult the FRC is determined by the balance between the ribcage and the lung's elasticity, being the resulting volume sometimes called elastic equilibrium volume, expiratory level or positive and expiratory pressure (intrinsic PEEP). In infants, this passive expiration results in a very low volume due to the ribcage's large compliance which practices a reduced distending pressure driven to the outside facilitating the collapse [13]-[16]. This difference in the mechanical respiration of adults compared to infants may eventually indicate against this technique in infants when they are breathing spontaneously.

The infants show, however, an ability to modulate the expiratory flow (using a combination of post-inspiratory diaphragmatic activity and laryngeal closure by vagal stimulation of the laryngeal adductor muscles that increase resistance during exhalation, increasing the variable of expiratory time) and expiratory time (by increasing the respiratory frequency and shortening the expiratory time) so that they breathe before the volume of elastic equilibrium is reached. These phenomena have as a result a dynamic increase in RR above the determined by the passive mechanical properties of his/her respiratory device [13]-[16]. The child's crying may also have contributed to the increase in the RR, because some children cried [2] during the maneuver and immediately after.

Postiaux [2] warns that respiratory physiotherapy can cause side effects which must be known as a possible deterioration of the vital signs in children that are intubated or not, inducing artificial ventilation and gastro esophageal reflux and that the therapist must be capable to assess the severity signs: respiratory rate, cyanosis, nutrition, difficulty breathing, fatigue, or respiratory failure.

The $\mathrm{SpO}_{2}$ also showed significant differences, being decreased in patients of the atelectasis group immediately after reducing furthermore 10 minutes after the MCC maneuver. This may have occurred because the alveolar surface of the child is smaller than that of adults. Regarding the correlation of $\mathrm{SpO}_{2}$ and $\mathrm{HR}$ after MCC it shows that the maintenance of $\mathrm{SpO}_{2}$ after the technique was applied was due to the increase in HR.

In each respiratory cycle small tidal volumes are generated, thus occurs the need for higher respiratory frequencies to maintain an adequate minute volume. With that, the high respiratory rate associated with low tidal volume has the disadvantages of high energy expenditure and also a greater "loss" of volume by ventilating areas of anatomic dead space more often, that truly are ventilated areas and that do not perform exchanges, such as the trachea and bronchi [15]-[18].

Another problem associated with the small alveolar surface lies in the fact that when children have compromised lung parenchyma, even if not very extensive, due to the sharp decline in the area of trade, are likely to have early hypoxemia [16], thus favoring the "desaturation" and hypoventilation.

The main limitation is not having compared the MCC with TILA, so future studies are needed although based in this study we do not recommend the use of MCC in infants.

\section{Conclusion}

The results obtained with the MCC technique in this study showed no significant hemodynamic changes, however, there were a decrease in $\mathrm{SpO}_{2}$, an increase of $\mathrm{RR}$ and a worsening of clinical signs of respiratory distress. Given this, one can consider that there are controversies about the benefits, mechanism of action, physiological and therapeutic effects of MCC when applied to infants. 


\section{References}

[1] Anonymous (1985) Acute Respiratory Infections in Under-Fives: 15 Millions Death a Year. The Lancet, 28, $699-701$.

[2] Postiaux, G. (2004) Fisioterapia Respiratória Pediátrica. O tratamento guiado pela ausculta pulmonar. Artmed, Porto Alegre.

[3] Gomes, E.L.F.D., Postiaux, G., Medeiros, D.R.L., Monteiro, K.K.D.S., Sampaio, L.M.M., Costa, D. (2012) Chest Physical Therapy Is Effective in Reduce Clinical Score in Bronchiolitis: Randomized Controlled Trial. Brazilian Journal of Physical Therapy, 16, 241-247. http://dx.doi.org/10.1590/S1413-35552012005000018

[4] Paludo, C., Zhang, L., Lincho, C.S., Lemos, D.V., Real, G.G. and Bergamin, J.A. (2008) Chest Physical Therapy for Children Hospitalised with Acute Pneumonia: A Randomised Controlled Trial. Thorax, 63, 791-794. http://dx.doi.org/10.1136/thx.2007.088195

[5] Johnston, C. and Carvalho, W.B. (2008) Atelectasia em pediatria: macanismos, diagnóstico e tratamento. Revista da Associação Médica Brasileira, 54, 455-460. http://dx.doi.org/10.1590/S0104-42302008000500021

[6] Perrota, C., Ortiz, Z. and Roque, M. (2005) Chest Physiotherapy for Acute Bronchiolitis in Paediatric Patients between 0 and 24 Months Old. Cochrane Database of Systematic Reviews, 2, CD004873.

[7] Sixel, B.S., Lemes, D.A., Pereira, K.A. and Guimarães, F.S. (2007) Compressão manual torácica em pacientes com insuficiência respiratória aguda. Fisioterapia Brasil, 8, 103-106.

[8] Lima J.G.M., Reis, L.F.F., Moura, F.M., Souza, C.P.V., Walchan, E.M. and Bergmann, A. (2008) Compressão manual torácica em um modelo experimental de atelectasia em ratos Wistar. Revista Fisioterapia em Movimento, 21, 77-82.

[9] Della Via, F., Oliveira, R.A.R.A. and Dragosavak, D. (2012) Effects of Manual Chest Compression and Descompression Maneuver on Lung Volumes, Capnography and Pulse Oxymeter in Patients Receiving Mechanical Ventilation. Brazilian Journal of Physical Therapy, 16, 354-359.

[10] Herri, S. (2007) Technique Insufflatoire de Levée d’Atélectasie (TILA) en réanimation néonatale. Kinésithérapie, la Revue, 65, 30-34.

[11] Postiaux, G., Ladha, K. and Lens, E. (1995) Proposition d’une kinésithérapie respiratoire confortée par l’équation de Rohrer. Application au nourrisson broncho-obstructif. Annales de Kinésithérapie, 22, 343-354.

[12] Sharp, J.T., Druz, W.S., Balagot, R.C., Baudelin, V.R., Danon, J. (1970) Total Respiratory Compliance in Infants and Children. Journal of Applied Physiology, 29, 775-779.

[13] Gerhardt, T. and Bancalari, E. (1980) Chest Wall Compliance in Full-Term and Premature Infants. Acta Paediatrica Scandinavica, 69, 359-364. http://dx.doi.org/10.1111/j.1651-2227.1980.tb07093.x

[14] Rabbette, P.S. and Stocks, J. (1998) Influence of Volume Dependency and Timing of Airway Occlusions of the Hering-Breuer Reflex in Infants. Journal of Applied Physiology, 85, 2033-2039.

[15] Hassan, A., Gossage, J., Ingram, D., Lee, S., Milner, A.D. (2001) Volume of Activation of the Hering-Breuer Inflation Reflex in the Newborn Infant. Journal of Applied Physiology, 90, 763-769.

[16] Davis, G.M., Coater, A.L., Papageorgiou, A. and Bureau, M.A. (1988) Direct Measurement of Static Chest Wall Compliance in Animal and Human Neonates. Journal of Applied Physiology, 65, 1093-1098.

[17] Schechter, M.S. (2007) Airway Clearance Aplications in Infants and Children. Respiratory Care, 52, 1382-1391.

[18] Saianda, A. and Bandeira, T. (2009) Estudo funcional respiratório do lactente-Estado da arte e aplicabilidade clínica. Acta Pediátrica Portuguesa, 40, 168-167. 